\title{
Intensive Cavity-Magnomechanical Cooling of a Levitated Macromagnet
}

\author{
A. Kani, ${ }^{*}$ B. Sarma®, and J. Twamley \\ Quantum Machines Unit, Okinawa Institute of Science and Technology Graduate University, Okinawa 904-0495, Japan
}

(Received 15 August 2021; accepted 8 December 2021; published 4 January 2022)

\begin{abstract}
We describe microwave cavity-magnomechanical center-of-mass cooling of a levitated magnetic sphere. The standing magnetic component of the electromagnetic wave within a microwave cavity exerts a dynamical force on a magnonic crystalline sphere and dissipates the mechanical energy through scattering into the magnon mode. The coupling is established by the magnetic dipole interaction and enriched by the collective spin motion. We find that the final cooled phonon occupation achieved is an intensive property independent of the mass and size of the sphere, in contrast to standard optomechanical couplings. This is of particular importance for testing quantum mechanics with macroscopic objects.
\end{abstract}

DOI: 10.1103/PhysRevLett.128.013602

Introduction.-Cooling massive systems to their motional quantum ground state is a long-standing goal, not only for observing quantum signatures in the macroscopic world [1-3], but also in performing ultrahigh-precision measurements $[4,5]$ to explore physics beyond the standard model [6], search for dark matter [7], understand gravitational decoherence [8,9], and toward marking the classicalquantum boundary [10]. Trapping and cooling a large particle, with diameters $d>\mu \mathrm{m}$, is highly challenging for a number of reasons. Standard optical trapping becomes difficult to operate for objects larger than a micron and only a few works have studied the optical levitation of large objects [11-14]. There are a variety of methods to motionally trap a magnetic particle, such as within an optical trap [15], magnetic trap [16,17], ion trap [2], or Paul trap [18], or can be clamped to an ultrahigh- $Q$ mechanical resonator $[19,20]$. Until now, almost all levitated motional cooling has been confined to nanometer-sized or femto- or nanogram massed objects [21-24]. Magnetic levitation, in particular, has the potential to levitate and motionally cool quite macroscopic objects [18,25-28]. Magnetic levitation can support $\sim$ millimeter-sized spherical magnets, either floating above a superconductor in free space [25] or within a microwave cavity [29]. Cooling of the trapped object crucially depends on the strength of the control fields one can exert and typically this single excitation coupling strength scales as $g \equiv \eta x_{\mathrm{ZPM}}$, where $\eta$ quantifies the coupling strength to the particle's position $x(t)$, and $x_{\mathrm{ZPM}}$ is the zero-point motion of the particle in the trap $x_{\mathrm{ZPM}} \sim \sqrt{\hbar / 2 m \omega_{c}}$, where $\omega_{c}$ is the center-of-mass

Published by the American Physical Society under the terms of the Creative Commons Attribution 4.0 International license. Further distribution of this work must maintain attribution to the author(s) and the published article's title, journal citation, and DOI. mechanical oscillation frequency. For large particles, the decrease in $x_{\mathrm{ZPM}}$ with mass leads to a greatly reduced coupling strength, making ground-state cooling hard to achieve.

Here, we propose a microwave cavity-magnomechanical system wherein the reduction of coupling strength with increasing mass is compensated for when the coupling to the particle's position is achieved via magnonic excitations, where a magnon is the quanta of collective spin excitation. We show that by including a magnonic element one can enrich the cavity-magnomechanical coupling strength by a factor of $\sqrt{n}$, where $n$ is the total number of spins in the particle. This yields a cooling rate that is nearly independent of the size and mass of the particle being cooled; i.e., the cooling rate becomes an intensive property of the particle. The $\sqrt{n}$ scaling is enormous in high-density solidstate spin systems [30-32]. Among them, yttrium iron garnet $-\mathrm{Y}_{3} \mathrm{Fe}_{5} \mathrm{O}_{12}$ (YIG) - has attracted considerable interest due to its intrinsic low magnetic damping [30,33]; it is a promising candidate for spintronic applications [34-36], quantum computing [37], and quantum memories [38]. YIG is a ferrimagnet with spin density $\sim 4.22 \times 10^{27} \mathrm{~m}^{-3}$ [33]. The high spin density of YIG has been exploited for strong and ultrastrong coupling of magnons to microwave cavity modes $[32,33,39]$ and microwave to optical photon conversion [40]. Here, we utilize the collective spin interaction to enhance the mechanical coupling of a macroscopic YIG sphere and effectively cool its motional degree of freedom to achieve phonon occupations that are 6 orders of magnitude smaller than standard cooling for large (millimeter)-sized particles.

Model.-We consider a YIG sphere whose center of mass is trapped in a harmonic potential inside a driven microwave cavity as shown in Fig. 1(a). A highly polished pure single-crystal YIG can support high-quality spin waves. Spin waves are the collective dynamics of the spin 


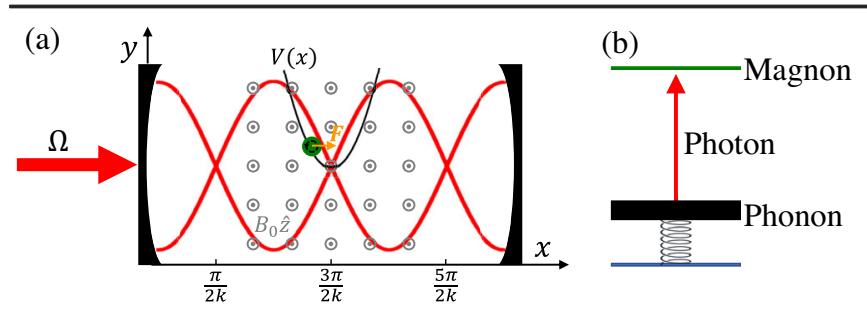

FIG. 1. Schematic of the system for microwavemagnomechanical intensive cooling: (a) A YIG sphere is trapped in a harmonic potential $V(x)$ at a node of the driven microwave cavity magnetic field $\boldsymbol{B}_{\text {cav }}=\hat{\boldsymbol{y}} B \cos (k x)$. A uniform bias magnetic field $B_{0} \hat{z}$ is applied to induce magnon. The cavity field gradient couples the magnon and the mechanical center-of-mass phonon. (b) The simplified cavity-magnomechanical resonator.

precession due to mutual interaction between the spins [41-44]. To induce precession, a large static homogeneous magnetic field $\left(\boldsymbol{B}=B_{0} \hat{z}\right)$, above the saturation magnetic field, is applied to align all the magnetic moments of the YIG sphere with the external $\boldsymbol{B}$ field. Because of the finite geometry, the magnetized sphere confines the spin wave and acts as a spin wave resonator. Unlike the electromagnetic wave resonators, the frequency of the magnon modes are independent of the size of the sphere, but can be tuned by varying $B_{0}$. The spatial profile of the confined modes do change with the sphere size.

We focus on the fundamental magnon mode (Kittel magnon), which is a uniform magnetization precession $\boldsymbol{m}=m(\hat{\boldsymbol{x}}+i \hat{\boldsymbol{y}}) e^{-i \omega_{b} t}+$ c.c. about the bias field $\boldsymbol{B}$ at the frequency $\omega_{b}=|\gamma| B_{0}$, where $m$ is the complex amplitude of the Kittel mode, and $\gamma=2 \pi \times 28 \mathrm{GHz} / \mathrm{T}$ is the gyromagnetic ratio. For a bias field $B_{0} \sim 1 \mathrm{~T}$, the Kittel magnon can resonantly interact with microwave magnetic field of wavelength $\sim 1 \mathrm{~cm}$. We consider the microwave cavity mode along $\hat{\boldsymbol{y}}$, which is of the form $\boldsymbol{B}_{\mathrm{cav}}=\hat{\boldsymbol{y}} B \cos (k x)$, to cool the center-of-mass $\hat{\boldsymbol{x}}$ degree of freedom of the YIG sphere, where $k$ is the microwave field wave number. For a YIG sphere size that is less than the cavity field wavelength, the coupling between the microwave cavity $\boldsymbol{B}_{\text {cav }}$ and the Kittel magnon can be well described by the magnetic dipole interaction $\mathcal{H}=-\boldsymbol{m} \cdot \boldsymbol{B}_{\text {cav }}$. The Kittel magnon frequency can be tuned to be near resonant with the cavity and we thus ignore the interactions of other magnon modes with the microwave cavity mode. Within the rotating wave approximation, when the cavity-magnon interaction is not ultrastrong, the total quantum mechanical Hamiltonian of the cavity-magnonic system can be written as

$$
\begin{aligned}
\mathcal{H} / \hbar= & \omega_{a} a^{\dagger} a+\omega_{b} b^{\dagger} b+g_{a b}\left(a b^{\dagger}+a^{\dagger} b\right) \cos (k x) \\
& +\Omega\left(a e^{i \omega_{l} t}+a^{\dagger} e^{-i \omega_{l} t}\right),
\end{aligned}
$$

where $a(b)$ is the bosonic operator for the cavity (magnon) mode at frequency $\omega_{a}\left(\omega_{b}\right)$, and $\Omega$ is a microwave cavity drive at frequency $\omega_{l}$. The coupling strength $g_{a b}$ between the cavity photon and the YIG magnon is given by [33]

$$
g_{a b}=\frac{\gamma}{2} \sqrt{\frac{\hbar \omega_{a} \mu_{0}}{V_{a}}} \sqrt{2 \rho_{s} V s},
$$

where $V_{a}$ and $V$ are the volumes of the cavity mode and the YIG sphere, respectively, $\mu_{0}$ is the vacuum permeability, $\rho_{s}$ is the spin density of the YIG sphere, and $s=5 / 2$ is the YIG's ground-state spin number. The coherent coupling $g_{a b}$ is enhanced due to the collective interaction of spins by a factor of the square root of the total number of spins. The high spin density of YIG leads to strong photon-magnon coupling ( $g_{a b}>$ both cavity and magnon damping rates), for the YIG sphere radius $R \geq 100 \mu \mathrm{m}[32,33,39]$. As the YIG sphere is trapped in a harmonic potential, we have to account for the motional degree of freedom in Eq. (1). For the YIG sphere with size $R>1 \mathrm{~nm}$ in a low-frequency trap $(\sim 50 \mathrm{kHz})$ and at room temperature, the thermal variance in the particle's position $\Delta x=\sqrt{k_{b} T /\left(m \omega^{2}\right)}$ [45] is much smaller than the microwave wavelength. Thus, expanding $\cos (k x)$ in Eq. (1) in a Taylor series up to first order in particle position around the position of the trap minimum $\left(x_{0}\right)$ yields a cavity-magnomechanical interaction Hamiltonian, in the frame rotating at the frequency $\omega_{l}$, as

$$
\begin{aligned}
\tilde{\mathcal{H}} / \hbar= & \Delta_{a} a^{\dagger} a+\Delta_{b} b^{\dagger} b+\omega_{c} c^{\dagger} c+g_{a b} \cos \left(k x_{0}\right)\left(a b^{\dagger}+a^{\dagger} b\right) \\
& -g_{a b c} \sin \left(k x_{0}\right)\left(a b^{\dagger}+a^{\dagger} b\right)\left(c+c^{\dagger}\right)+\Omega\left(a+a^{\dagger}\right),
\end{aligned}
$$

where $\Delta_{j}=\omega_{j}-\omega_{l}$ is the detuning and $c$ is the bosonic operator for the center-of-mass oscillation at the trap frequency $\omega_{c}$. The three-body coupling strength is given by

$$
g_{a b c}=g_{a b} k x_{\mathrm{ZPM}}=g_{a b} k \sqrt{\frac{\hbar}{2 \rho_{m} V \omega_{c}}},
$$

where $\rho_{m}=5170 \mathrm{~kg} \mathrm{~m}^{-3}$ is the mass density of the YIG sphere. Although the smallness of the zero-point motion $\left(x_{\mathrm{ZPM}}\right)$ of the massive particle weakens the coupling, due to collective spin interaction the cavity-magnomechanical coupling strength $g_{a b c}$ is size independent, i.e., intensive. This is in contrast to the standard optomechanical coupling and this size independence makes this coupling highly suitable for cooling massive mechanical oscillators. The above Hamiltonian includes the interaction of the magnon with the cavity field $\boldsymbol{B}_{\text {cav }}\left(x_{0}\right)$, as well as the magnomechanical interaction driven by the cavity field. By choosing an appropriate position $x_{0}$, one can tune between either type of these interactions. Strong cavity-magnon interaction can be engineered by placing the trap at an antinode of the cavity magnetic field [32,33,39]. Instead, to cool the mechanical motion, we optimize the 
cavity-magnomechanical coupling rate by positioning the trap minimum to be at a node of the cavity magnetic field $\left[k x_{0}=(2 n+1) \pi / 2\right]$, where the field gradient is maximum.

Dynamics of cooling.-The dynamics of the coupled system is described by the quantum master equation, which reads

$\dot{\rho}=\frac{1}{i \hbar}[\tilde{\mathcal{H}}, \rho]-\sum_{j=a, b, c} \frac{\gamma_{j}}{2}\left[\left(\bar{n}_{j}+1\right) \mathcal{L}[j] \rho+\bar{n}_{j} \mathcal{L}\left[j^{\dagger}\right] \rho\right]$,

where $\rho$ is the density operator, $\gamma_{j}$ is the damping rate, and $\bar{n}_{j}=\left(\exp \left[\hbar \omega_{j} / k_{B} T\right]-1\right)^{-1}$ is the mean thermal occupation of the bosonic mode $j \in\{a, b, c\}$. Here, $T$ is the temperature of the thermal environment, $k_{B}$ is the Boltzmann constant, and $\mathcal{L}[o] \rho=\left(o^{\dagger} o \rho+\rho o^{\dagger} o-\right.$ $\left.2 o \rho o^{\dagger}\right) / 2$ are the Lindblad superoperators accounting for dissipation and thermal fluctuations. Superconducting microwave cavities possess an extremely high-quality factor exceeding $10^{11}$ [46,47], and only a small input microwave power $P \sim-22 \mathrm{~dB} \mathrm{~m}$ is sufficient to achieve the cooling studied below. The damping of the mechanical motion of the levitated particle is primarily due to background gas friction and can be tuned by varying the environmental pressure. Practically, a nanomechanical oscillator with $Q=10^{8}$ has been reported at a pressure of $P=0.5 \times 10^{-6} \mathrm{mbar}$ [48]. In an ultrahigh vacuum with $P \sim 10^{-10}$ mbar, it has been estimated that $Q>10^{12}$ [49], and it is even better for a macroscopic particle as the quality factor scales linearly with particle size [50]. In YIG, the loss rate of the magnon mode can be $\gamma_{b} / 2 \pi<\mathrm{MHz}$ [51]. Considering ultrapure YIG, even smaller linewidths are possible.

From the definition of the density matrix, the expectation value of an operator is $\langle o\rangle=\operatorname{Tr}[\rho o]$, and from the master equation, the time evolution of the bosonic operators, including the thermal fluctuation over and above the mean values, can be written as

$\dot{a}=-\left(\frac{\gamma_{a}}{2}+i \Delta_{a}\right) a-i g_{a b c} b\left(c^{\dagger}+c\right)-i \Omega+\sqrt{\gamma_{a}} \xi_{a}$,

$\dot{b}=-\left(\frac{\gamma_{b}}{2}+i \Delta_{b}\right) b-i g_{a b c} a\left(c^{\dagger}+c\right)+\sqrt{\gamma_{b}} \xi_{b}$,

$\dot{c}=-\left(\frac{\gamma_{c}}{2}+i \omega_{c}\right) c-i g_{a b c}\left(a^{\dagger} b+a b^{\dagger}\right)+\sqrt{\gamma_{c}} \xi_{c}$,

where $\xi_{j}$ is the stochastic noise operator due to thermal baths that obeys $\left\langle\xi_{j}(t)\right\rangle=0,\left\langle\xi_{j}(t) \xi_{j}^{\dagger}\left(t^{\prime}\right)\right\rangle=\left(\bar{n}_{j}+1\right) \delta\left(t-t^{\prime}\right)$, and $\left\langle\xi_{j}^{\dagger}(t) \xi_{j}\left(t^{\prime}\right)\right\rangle=\bar{n}_{j} \delta\left(t-t^{\prime}\right)$. Equation (4) is the nonlinear quantum Langevin equation (QLE). As can be read from Eq. (4), the photon and magnon are exchanged through mechanical coupling, which can deplete the mechanical energy.
We now consider the cavity to be driven by a strong microwave field, but not into the multistable regime. The steady-state solution of the noiseless QLE is

$$
a_{0}=-\frac{2 \Omega}{i \gamma_{a}-2 \Delta_{a}}, \quad b_{0}=c_{0}=0
$$

The cooling efficiency of the standard sideband cooling of a massive oscillator requiring strong drive is mainly limited by internal heating from absorption [24,52]. However, in our scheme, no photon is directly diverted into exciting magnons, on average, in the YIG $\left(b_{0}=0\right)$. Even though the magnon frequency is near resonant with the cavity mode, trapping the particle at the node of the cavity magnetic field, where $g_{a b}=0$, prevents direct absorption of microwave photons and prevents heating of the bulk magnonic crystal. The steady-state solution is independent of the coupling $g_{a b c}$. Only the fluctuations around the steady state experience the cavity-magnomechanical coupling. Considering these fluctuations to be small, the dynamics about the steady state are characterized by linearizing the QLE around the equilibrium value $\left[a=a_{0}+\tilde{a}(t), b=\right.$ $\left.b_{0}+\tilde{b}(t), c=c_{0}+\tilde{c}(t)\right]$. The linearized QLE for the quantum noise operators are

$$
\begin{aligned}
& \dot{\tilde{a}}=-\left(\frac{\gamma_{a}}{2}+i \Delta_{a}\right) \tilde{a}+\sqrt{\gamma_{a}} \xi_{a}, \\
& \dot{\tilde{b}}=-\left(\frac{\gamma_{b}}{2}+i \Delta_{b}\right) \tilde{b}-i G\left(\tilde{c}^{\dagger}+\tilde{c}\right)+\sqrt{\gamma_{b}} \xi_{b}, \\
& \dot{\tilde{c}}=-\left(\frac{\gamma_{c}}{2}+i \omega_{c}\right) \tilde{c}-i G\left(\tilde{b}+\tilde{b}^{\dagger}\right)+\sqrt{\gamma_{c}} \xi_{c},
\end{aligned}
$$

where $G=\left|a_{0}\right| g_{a b c}$ is the cavity-enhanced effective coupling strength. Here, we have ignored the phase of $a_{0}$ to define $G$ as a real positive number. In Eq. (5), the fluctuation of the cavity field is decoupled from rest of the system. The three-body cavity-magnomechanical system reduces into an effective two-body magnomechanical system coupled by the strong coherent cavity field $a_{0}$. To increase $a_{0}$ we can drive the microwave cavity at resonance $\omega_{l}=\omega_{a}$ and maximize the magnon-phonon coupling strength $G$. This linearized QLE (5) corresponds to a Hamiltonian of the form

$\frac{\mathcal{H}_{L}}{\hbar}=\Delta_{a} \tilde{a}^{\dagger} \tilde{a}+\Delta_{b} \tilde{b}^{\dagger} \tilde{b}+\omega_{c} \tilde{c}^{\dagger} \tilde{c}-G\left(\tilde{b}^{\dagger}+\tilde{b}\right)\left(\tilde{c}+\tilde{c}^{\dagger}\right)$.

To analyze the system further, we solve Eq. (5) in the frequency domain and then, by using the properties of the quantum noise operators $\xi(\omega)$, we obtain the power 

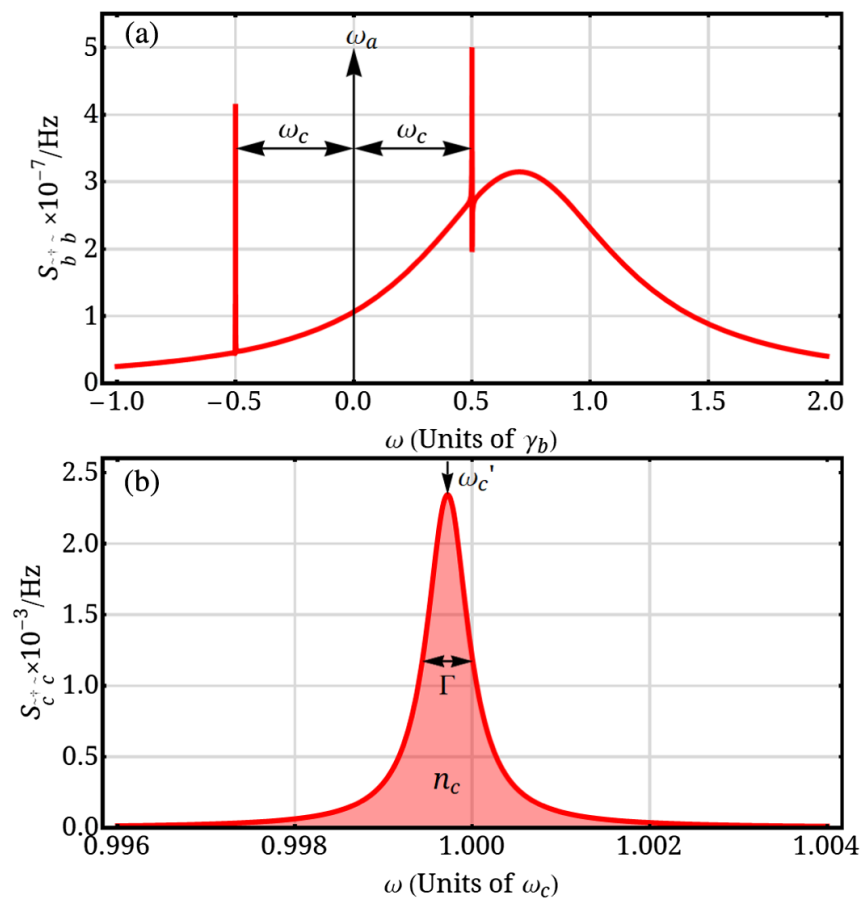

FIG. 2. Power spectra of the coupled systems: (a) Magnon and (b) mechanical power spectral density. The parameters are $\left(\gamma_{b}, \gamma_{c}, \omega_{b}, \omega_{c}, \Delta_{b}, G\right) / 2 \pi=\left(0.1 \mathrm{MHz}, 10^{-6} \mathrm{~Hz}, 30 \mathrm{GHz}, 50 \mathrm{kHz}\right.$, $70 \mathrm{kHz}, 1 \mathrm{kHz}$ ), and the background temperature $T=1 \mathrm{~K}$ with the mean thermal magnon and phonon occupancies as, respectively, $\bar{n}_{b}=0.31$ and $\bar{n}_{c}=4.17 \times 10^{5}$.

spectral density $S_{j^{\dagger} j}(\omega)=\int_{-\infty}^{\infty} j^{\dagger}(\omega) j\left(\omega^{\prime}\right) d \omega^{\prime}$ of the bosonic modes to characterize the system. The steady-state power spectrum of the magnon, as well as the mechanical mode, are shown in Fig. 2. The sidebands in the magnon spectrum, at frequencies $\omega=\omega_{l} \pm \omega_{c}$, are generated by the mechanical coupling driven by the cavity photon at the frequency $\omega_{l}$. From the mechanical power spectrum [Fig. 2(b)], the center-of-mass oscillation is characterized by measuring the peak position $\omega_{c}^{\prime}$, the spectral width $\Gamma$, and the area under the spectrum $n_{c}$, and they are all shown in Fig. 3. The magnomechanical coupling induces a shift in the mechanical resonance frequency that is relatively small for weak coupling. The spectral width reflects the effective damping or cooling rate of the mechanical motion, which includes additional damping due to net phonon scattering into the magnon mode and describes how fast the system reaches the steady state. The area under the spectrum is the steady-state mean phonon occupancy. We find that there is an optimal detuning $\Delta_{b}^{*}$ for which the steady-state cooled phonon occupancy is minimal.

Optimizing the cooling.-Now we describe the dynamics of the cooling process and find optimal parameters to reach the lowest phonon occupations. By using the linearized Hamiltonian (6) in the master equation (3), one can obtain the following set of coupled differential equations for the time evolution of the expectation value of the second-order moments:

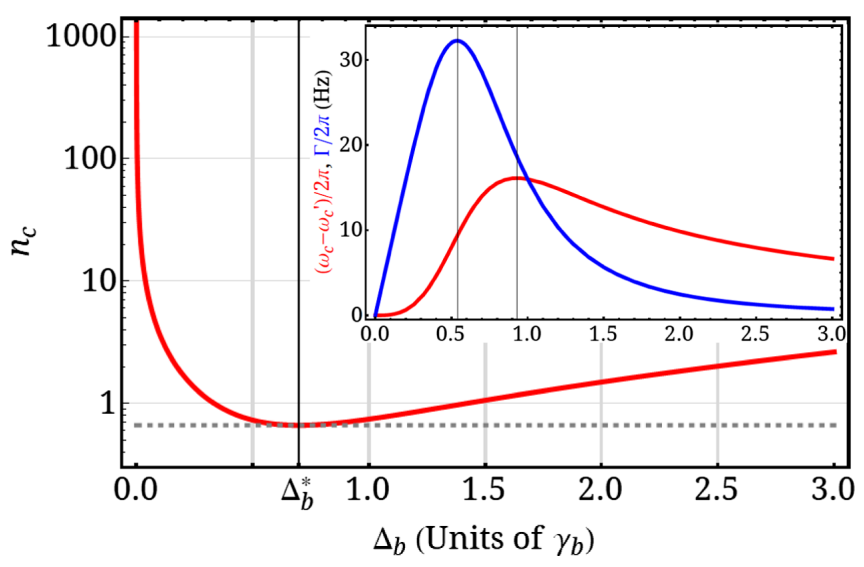

FIG. 3. Mechanical oscillator's properties: Mean final phonon occupancy as a function of magnon detuning. Inset shows the induced mechanical frequency shift (red), as well as effective cooling rate (blue). Other parameters are the same as in Fig. 2.

$$
\begin{aligned}
\left\langle\tilde{b}^{\dagger} \tilde{\tilde{b}}\right\rangle= & -\gamma_{b}\left\langle\tilde{b}^{\dagger} \tilde{b}\right\rangle+i G\left(\langle\tilde{b} \tilde{c}\rangle+\left\langle\tilde{b} \tilde{c}^{\dagger}\right\rangle-\text { c.c. }\right)+\gamma_{b} \bar{n}_{b}, \\
\left\langle\tilde{c}^{\dagger} \tilde{c}\right\rangle= & -\gamma_{c}\left\langle\tilde{c}^{\dagger} \tilde{c}\right\rangle+i G\left(\langle\tilde{b} \tilde{c}\rangle+\left\langle\tilde{b}^{\dagger} \tilde{c}\right\rangle-\text { c.c. }\right)+\gamma_{c} \bar{c}_{c}, \\
\langle\tilde{b} \dot{\tilde{c}}\rangle= & -\left(\frac{1}{2}\left(\gamma_{b}+\gamma_{c}\right)+i\left(\Delta_{b}+\omega_{c}\right)\right)\langle\tilde{b} \tilde{c}\rangle \\
& -i G\left(\left\langle\tilde{b}^{\dagger} \tilde{b}\right\rangle+\langle\tilde{b} \tilde{b}\rangle+\left\langle\tilde{c}^{\dagger} \tilde{c}\right\rangle+\langle\tilde{c} \tilde{c}\rangle+1\right), \\
\left\langle\tilde{b} \tilde{c^{\dagger}}\right\rangle= & -\left(\frac{1}{2}\left(\gamma_{b}+\gamma_{c}\right)+i\left(\Delta_{b}-\omega_{c}\right)\right)\left\langle\tilde{b} \tilde{c}^{\dagger}\right\rangle \\
& +i G\left(\left\langle\tilde{b}^{\dagger} \tilde{b}\right\rangle+\langle\tilde{b} \tilde{b}\rangle-\left\langle\tilde{c}^{\dagger} \tilde{c}\right\rangle-\left\langle\tilde{c}^{\dagger} \tilde{c}^{\dagger}\right\rangle\right), \\
\langle\tilde{b} \tilde{\tilde{b}}\rangle= & -\left(\gamma_{b}+2 i \Delta_{b}\right)\langle\tilde{b} \tilde{b}\rangle-2 i G\left(\left\langle\tilde{b} \tilde{c}^{\dagger}\right\rangle+\langle\tilde{b} \tilde{c}\rangle\right), \\
\langle\tilde{c} \tilde{\tilde{c}}\rangle= & -\left(\gamma_{c}+2 i \omega_{c}\right)\langle\tilde{c} \tilde{c}\rangle-2 i G\left(\left\langle\tilde{b}^{\dagger} \tilde{c}\right\rangle+\langle\tilde{b} \tilde{c}\rangle\right) .
\end{aligned}
$$

We solve the above equations numerically with the initial conditions $\left\langle\tilde{b}^{\dagger} \tilde{b}\right\rangle(t=0)=\bar{n}_{b}$ and $\left\langle\tilde{c}^{\dagger} \tilde{c}\right\rangle(t=0)=\bar{n}_{c}$. The time evolution of the phonon occupancy is shown in Fig. 4, for different coupling strengths at the optimal cooling detuning point $\Delta_{b}^{*}$. Increasing $G$ improves the cooling rate. However, there is an optimal coupling value of $G^{*}$ for which the steady-state phonon occupancy is minimum. In Fig. 4, we also plot the steady-state mean phonon occupancy as a function of $G$. The optimal $G^{*}$ is well within the weak coupling regime $G<\omega_{c}, \gamma_{b}$. For the parameters considered here, the optimal cooling temperature reached is $T_{\text {eff }}=2.58 \mu \mathrm{K}$ with the mean phonon occupancy $n_{c}=$ 0.651 (parameters the same as in Fig. 2). This value is independent of the size of the YIG sphere. In Fig. 5, we compare the intensive cooling with the optomechanical cooling assisted by radiation pressure induced coupling $\mathcal{H}_{\text {int }}=\hbar g a^{\dagger} a\left(c+c^{\dagger}\right)$ for varying particle size with the same intracavity power. As long as the particle is much smaller than the microwave wavelength $(=1 \mathrm{~cm})$, the intensive cooling scheme allows us to reach the quantum 


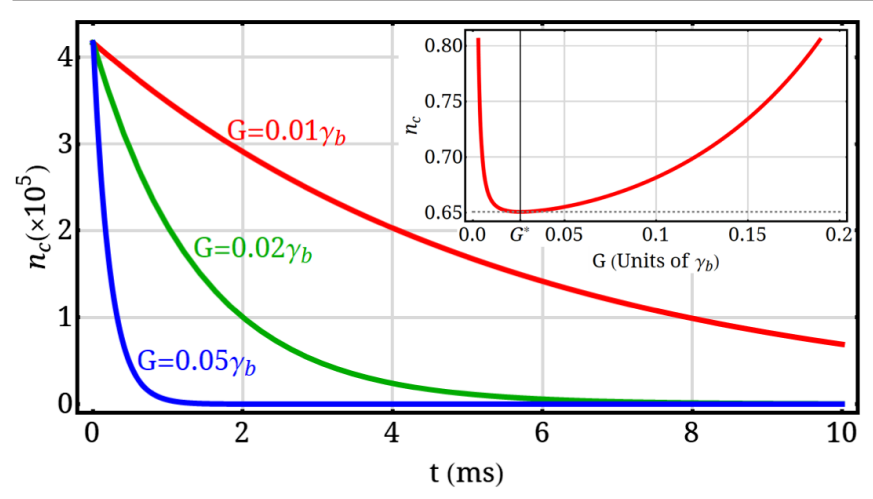

FIG. 4. Dynamics of the cooling process: Time evolution of the mechanical phonon occupancy for different magnomechanical coupling strengths $G$ (in units of phonon damping rate $\gamma_{b}$ ), at the optimal cooling detuning $\Delta_{b}^{*}$. The inset shows the steady-state phonon occupancy as a function of $G$ at $\Delta_{b}^{*}$. Other parameters are the same as in Fig. 2.

ground state, which is highly challenging with standard techniques.

In the limit $\gamma_{c} / \omega_{c} \ll 1$, and for weak coupling, the steady-state mean phonon occupancy is given by

$$
n_{c} \approx \frac{\gamma_{c} \bar{n}_{c}+4 \gamma_{b} G^{2}\left[\frac{\bar{n}_{b}+\frac{G^{2}\left(2 \bar{n}_{b}+1\right)}{2 \omega_{c}^{c}}}{\gamma_{b}^{2}+4\left(\Delta_{b}-\omega_{c}\right)^{2}}+\frac{\left(\bar{n}_{b}+1\right)-\frac{G^{2}\left(2 \bar{n}_{b}+1\right)}{2 \omega_{c}^{2}}}{\gamma_{b}^{2}+4\left(\Delta_{b}+\omega_{c}\right)^{2}}\right]}{\gamma_{c}+\left[\frac{4 \gamma_{b} G^{2}}{\gamma_{b}^{2}+4\left(\Delta_{b}-\omega_{c}\right)^{2}}-\frac{4 \gamma_{b} G^{2}}{\gamma_{b}^{2}+4\left(\Delta_{b}+\omega_{c}\right)^{2}}\right]} .
$$

As it is apparent in the above equation [53-55], the incoherent damping $\Gamma_{-}$and heating $\Gamma_{+}$rates due to phonon scattering into the magnon sidebands $\omega_{l}+\omega_{c}$ and $\omega_{l}-\omega_{c}$ are

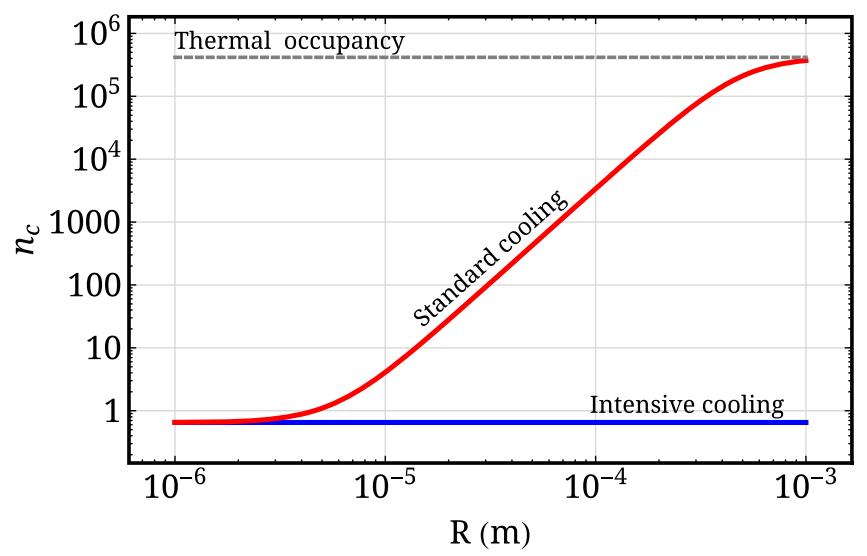

FIG. 5. Comparison between the standard radiation pressure cooling and the intensive cooling as a function of sphere radius at the optimal cooling detuning point for the same intracavity power.
$\Gamma_{ \pm}=4 \gamma_{b} G^{2}\left[\frac{\bar{n}_{b} \pm \frac{G^{2}\left(2 \bar{n}_{b}+1\right)}{2 \omega_{c}^{2}}}{\gamma_{b}^{2}+4\left(\Delta_{b} \mp \omega_{c}\right)^{2}}+\frac{\left(\bar{n}_{b}+1\right) \mp \frac{G^{2}\left(2 \bar{n}_{b}+1\right)}{2 \omega_{c}^{2}}}{\gamma_{b}^{2}+4\left(\Delta_{b} \pm \omega_{c}\right)^{2}}\right]$.

These are the rates at which the energies are being exchanged between the magnonic and the mechanical system through the cavity mode. The net damping rate of the mechanical motion is given by $\Gamma=\gamma_{c}+\left(\Gamma_{-}-\Gamma_{+}\right)$. Having large $\Gamma \gg \gamma_{c}$ reduces the thermal phonon occupancy from $\bar{n}_{c}$ to $\bar{n}_{c} \gamma_{c} / \Gamma$. However, there is also an additional noise from the magnon scattering that heats up the mechanical motion and increases the phonon occupancy by an amount of $\Gamma_{+} / \Gamma$. One can optimize this heating effect at the detuning $\Delta_{b}^{*}=\frac{1}{2} \sqrt{\gamma_{b}^{2}+4 \omega_{c}^{2}}$. Further, we can enhance $\Gamma$ by increasing $G$, but this will also increase $\Gamma_{+}$. As a result of these two competing effects, there will be an optimal coupling value of $G^{*}=\left\{\bar{n}_{c} \gamma_{b} \gamma_{c} \omega_{c} \Delta_{b}^{*} /\left[2\left(2 \bar{n}_{b}+1\right)\right]\right\}^{1 / 4}$, for which one can achieve a steady-state mean phonon occupancy of

$n_{c}=-\frac{1}{2}+\frac{\left(2 \bar{n}_{b}+1\right) \Delta_{b}^{*}}{2 \omega_{c}}+\sqrt{\frac{\bar{n}_{c} \gamma_{b} \gamma_{c}\left(2 \bar{n}_{b}+1\right) \Delta_{b}^{*}}{2 \omega_{c}^{3}}}$.

From Eq. (8), one can obtain the essential conditions for ground-state cooling. This cooling scheme demands low thermal magnon occupancy $\bar{n}_{b}<1$. As the magnon frequency is in the microwave regime, ground-state cooling can be reached by precooling the system to $\bar{n}_{b}<1$. In Fig. 5, we plot the performance of our intensive scheme vs normal radiation pressure cooling. For the latter, the optomechanical coupling $g_{\text {om }} \propto x_{\mathrm{ZPM}} \propto 1 / \sqrt{m} \sim R^{-3 / 2}$, leading to a severe inability to cool larger spheres, while our intensive scheme has a coupling $g_{a b c}$, which is independent of $R$, as the reduction in the zero-point length is exactly compensated by an increase in the spin number. At $T=300 \mathrm{~K}\left(\bar{n}_{b}=207.882\right)$, the final optimal phonon occupancy achieved by our intensive scheme can be as low as $n_{c} \sim 295$. To achieve ground-state cooling $n_{c}<1$, one must have a mechanical frequency $\omega_{c}>\gamma_{b} / \sqrt{32}$, which is essentially a resolved sideband condition.

Conclusions.-We have proposed and analyzed cavitymagnomechanical coupling for intensive ground-state cooling of a levitated YIG sphere. For an ultrahigh- $Q$ mechanical oscillation, we have derived the final occupancy of the mechanical mode and provided the essential conditions for optimal ground-state cooling. For a particle size that is much smaller than the microwave field wavelength, this cooling scheme is size independent and is consistent for a subcentimeter-sized particle. This three-body intensive cooling scheme can provide a route toward the preparation of massive motional quantum superpositions.

This work was supported by the Okinawa Institute of Science and Technology Graduate University. 
"KaniMohamed@oist.jp

[1] O. Romero-Isart, A. C. Pflanzer, F. Blaser, R. Kaltenbaek, N. Kiesel, M. Aspelmeyer, and J. I. Cirac, Large Quantum Superpositions and Interference of Massive NanometerSized Objects, Phys. Rev. Lett. 107, 020405 (2011).

[2] A. T.M. A. Rahman, Large spatial Schrödinger cat state using a levitated ferrimagnetic nanoparticle, New J. Phys. 21, 113011 (2019).

[3] P. Sekatski, M. Aspelmeyer, and N. Sangouard, Macroscopic Optomechanics from Displaced Single-Photon Entanglement, Phys. Rev. Lett. 112, 080502 (2014).

[4] B.P. Abbott et al., GW150914: The Advanced LIGO Detectors in the Era of First Discoveries, Phys. Rev. Lett. 116, 131103 (2016).

[5] J. Prat-Camps, C. Teo, C. C. Rusconi, W. Wieczorek, and O. Romero-Isart, Ultrasensitive Inertial and Force Sensors with Diamagnetically Levitated Magnets, Phys. Rev. Applied 8, 034002 (2017).

[6] D. C. Moore and A. A. Geraci, Searching for new physics using optically levitated sensors, Quantum Sci. Technol. 6, 014008 (2021).

[7] F. Monteiro, G. Afek, D. Carney, G. Krnjaic, J. Wang, and D. C. Moore, Search for Composite Dark Matter with Optically Levitated Sensors, Phys. Rev. Lett. 125, 181102 (2020).

[8] A. Bassi, A. Großardt, and H. Ulbricht, Gravitational decoherence, Classical Quantum Gravity 34, 193002 (2017).

[9] M. P. Blencowe, Effective Field Theory Approach to Gravitationally Induced Decoherence, Phys. Rev. Lett. 111, 021302 (2013).

[10] P. Bertet, S. Osnaghi, A. Rauschenbeutel, G. Nogues, A. Auffeves, M. Brune, J. M. Raimond, and S. Haroche, A complementarity experiment with an interferometer at the quantum-classical boundary, Nature (London) 411, 166 (2001).

[11] A. Ashkin and J. M. Dziedzic, Optical levitation by radiation pressure, Appl. Phys. Lett. 19, 283 (1971).

[12] F. Monteiro, W. Li, G. Afek, C. L. Li, M. Mossman, and D. C. Moore, Force and acceleration sensing with optically levitated nanogram masses at microkelvin temperatures, Phys. Rev. A 101, 053835 (2020).

[13] G. Guccione, M. Hosseini, S. Adlong, M. T. Johnsson, J. Hope, B. C. Buchler, and P. K. Lam, Scattering-Free Optical Levitation of a Cavity Mirror, Phys. Rev. Lett. 111, 183001 (2013).

[14] Y. Michimura, Y. Kuwahara, T. Ushiba, N. Matsumoto, and M. Ando, Optical levitation of a mirror for reaching the standard quantum limit, Opt. Express 25, 13799 (2017).

[15] T. Seberson, P. Ju, J. Ahn, J. Bang, T. Li, and F. Robicheaux, Simulation of sympathetic cooling an optically levitated magnetic nanoparticle via coupling to a cold atomic gas, J. Opt. Soc. Am. B 37, 3714 (2020).

[16] C. C. Rusconi, V. Pöchhacker, K. Kustura, J. I. Cirac, and O. Romero-Isart, Quantum Spin Stabilized Magnetic Levitation, Phys. Rev. Lett. 119, 167202 (2017).

[17] J. Gieseler, A. Kabcenell, E. Rosenfeld, J. D. Schaefer, A. Safira, M. J. A. Schuetz, C. Gonzalez-Ballestero, C. C. Rusconi, O. Romero-Isart, and M. D. Lukin, Single-Spin Magnetomechanics with Levitated Micromagnets, Phys. Rev. Lett. 124, 163604 (2020).
[18] P. Huillery, T. Delord, L. Nicolas, M. Van Den Bossche, M. Perdriat, and G. Hétet, Spin mechanics with levitating ferromagnetic particles, Phys. Rev. B 101, 134415 (2020).

[19] A. Vinante, G. Wijts, O. Usenko, L. Schinkelshoek, and T. H. Oosterkamp, Magnetic resonance force microscopy of paramagnetic electron spins at millikelvin temperatures, Nat. Commun. 2, 572 (2011).

[20] R. Fischer, D. P. McNally, C. Reetz, G. G. T. Assumpção, T. Knief, Y. Lin, and C.A. Regal, Spin detection with a micromechanical trampoline: Towards magnetic resonance microscopy harnessing cavity optomechanics, New J. Phys. 21, 043049 (2019).

[21] J. Millen and B. A. Stickler, Quantum experiments with microscale particles, Contemp. Phys. 61, 155 (2020).

[22] M. Rademacher, J. Millen, and Y. L. Li, Quantum sensing with nanoparticles for gravimetry: When bigger is better, Adv. Opt. Technol. 9, 227 (2020).

[23] U. Delić, M. Reisenbauer, K. Dare, D. Grass, V. Vuletić, N. Kiesel, and M. Aspelmeyer, Cooling of a levitated nanoparticle to the motional quantum ground state, Science $\mathbf{3 6 7}$, 892 (2020).

[24] J. Chan, T.P. M. Alegre, A. H. Safavi-Naeini, J. T. Hill, A. Krause, S. Gröblacher, M. Aspelmeyer, and O. Painter, Laser cooling of a nanomechanical oscillator into its quantum ground state, Nature (London) 478, 89 (2011).

[25] C. Timberlake, G. Gasbarri, A. Vinante, A. Setter, and H. Ulbricht, Acceleration sensing with magnetically levitated oscillators above a superconductor, Appl. Phys. Lett. 115, 224101 (2019).

[26] M.C. O'Brien, S. Dunn, J.E. Downes, and J. Twamley, Magneto-mechanical trapping of micro-diamonds at low pressures, Appl. Phys. Lett. 114, 053103 (2019).

[27] X. Jiang, J. Rudge, and M. Hosseini, Superconducting levitation of a mg-scale cavity mirror, Appl. Phys. Lett. 116, 244103 (2020).

[28] Y. Leng, R. Li, X. Kong, H. Xie, D. Zheng, P. Yin, F. Xiong, T. Wu, C.-K. Duan, Y. Du, Z.-q. Yin, P. Huang, and J. Du, Mechanical Dissipation below $1 \mu \mathrm{Hz}$ with a Cryogenic Diamagnetic Levitated Micro-Oscillator, Phys. Rev. Applied 15, 024061 (2021).

[29] N. K. Raut, J. Miller, J. Pate, R. Chiao, and J. E. Sharping, Meissner levitation of a millimeter-size neodymium magnet within a superconducting radio frequency cavity, IEEE Trans. Appl. Supercond. 31, 1 (2021).

[30] A. A. Serga, A. V. Chumak, and B. Hillebrands, YIG magnonics, J. Phys. D 43, 264002 (2010).

[31] H. Maier-Flaig, M. Harder, S. Klingler, Z. Qiu, E. Saitoh, M. Weiler, S. Geprägs, R. Gross, S. T. B. Goennenwein, and H. Huebl, Tunable magnon-photon coupling in a compensating ferrimagnet-from weak to strong coupling, Appl. Phys. Lett. 110, 132401 (2017).

[32] J. T. Hou and L. Liu, Strong Coupling between Microwave Photons and Nanomagnet Magnons, Phys. Rev. Lett. 123, 107702 (2019).

[33] X. Zhang, C.-L. Zou, L. Jiang, and H.X. Tang, Strongly Coupled Magnons and Cavity Microwave Photons, Phys. Rev. Lett. 113, 156401 (2014). 
[34] D. Hou, Z. Qiu, J. Barker, K. Sato, K. Yamamoto, S. Vélez, J. M. Gomez-Perez, L. E. Hueso, F. Casanova, and E. Saitoh, Tunable Sign Change of Spin Hall Magnetoresistance in Pt/NiO/YIG Structures, Phys. Rev. Lett. 118, 147202 (2017).

[35] G. Siegel, M. C. Prestgard, S. Teng, and A. Tiwari, Robust longitudinal spin-Seebeck effect in Bi-YIG thin films, Sci. Rep. 4, 4429 (2015).

[36] D. Qu, S. Y. Huang, J. Hu, R. Wu, and C. L. Chien, Intrinsic Spin Seebeck Effect in Au/YIG, Phys. Rev. Lett. 110, 067206 (2013).

[37] K. Pradeep Kumar, Single-qubit quantum gates using magnon-photon interaction, in Proceedings of the International Conference on Quantum Information (OSA, Washington, DC, 2011), p. QMI36.

[38] X. Zhang, C.-L. Zou, N. Zhu, F. Marquardt, L. Jiang, and H. X. Tang, Magnon dark modes and gradient memory, Nat. Commun. 6, 8914 (2015).

[39] Y. Tabuchi, S. Ishino, T. Ishikawa, R. Yamazaki, K. Usami, and Y. Nakamura, Hybridizing Ferromagnetic Magnons and Microwave Photons in the Quantum Limit, Phys. Rev. Lett. 113, 083603 (2014).

[40] Y.S. Ihn, S.-Y. Lee, D. Kim, S. H. Yim, and Z. Kim, Coherent multimode conversion from microwave to optical wave via a magnon-cavity hybrid system, Phys. Rev. B 102, 064418 (2020).

[41] A. Prabhakar and D. D. Stancil, Spin Waves (Springer, Boston, MA, 2009).

[42] D. D. Stancil, Theory of Magnetostatic Waves (Springer, New York, NY, 1993).

[43] C. Gonzalez-Ballestero, D. Hümmer, J. Gieseler, and O. Romero-Isart, Theory of quantum acoustomagnonics and acoustomechanics with a micromagnet, Phys. Rev. B 101, 125404 (2020).

[44] D. L. Mills, Quantum theory of spin waves in finite samples, J. Magn. Magn. Mater. 306, 16 (2006).
[45] T. Li, S. Kheifets, D. Medellin, and M. G. Raizen, Measurement of the instantaneous velocity of a Brownian particle, Science 328, 1673 (2010).

[46] S. Kuhr, S. Gleyzes, C. Guerlin, J. Bernu, U. B. Hoff, S. Deléglise, S. Osnaghi, M. Brune, J.-M. Raimond, S. Haroche, E. Jacques, P. Bosland, and B. Visentin, Ultrahigh finesse Fabry-Pérot superconducting resonator, Appl. Phys. Lett. 90, 164101 (2007).

[47] A. Romanenko, R. Pilipenko, S. Zorzetti, D. Frolov, M. Awida, S. Belomestnykh, S. Posen, and A. Grassellino, Three-Dimensional Superconducting Resonators at $T<$ $20 \mathrm{mK}$ with Photon Lifetimes up to $\tau=2 \mathrm{~s}$, Phys. Rev. Applied 13, 034032 (2020).

[48] J. Gieseler, L. Novotny, and R. Quidant, Thermal nonlinearities in a nanomechanical oscillator, Nat. Phys. 9, 806 (2013).

[49] D. E. Chang, C. A. Regal, S. B. Papp, D. J. Wilson, J. Ye, O. Painter, H. J. Kimble, and P. Zoller, Cavity opto-mechanics using an optically levitated nanosphere, Proc. Natl. Acad. Sci. U.S.A. 107, 1005 (2010).

[50] M. Imboden and P. Mohanty, Dissipation in nanoelectromechanical systems, Phys. Rep. 534, 89 (2014).

[51] X. Zhang, C.-L. Zou, L. Jiang, and H. X. Tang, Cavity magnomechanics, Sci. Adv. 2, e1501286 (2016).

[52] L. Qiu, I. Shomroni, P. Seidler, and T. J. Kippenberg, Laser Cooling of a Nanomechanical Oscillator to Its Zero-Point Energy, Phys. Rev. Lett. 124, 173601 (2020).

[53] I. Wilson-Rae, N. Nooshi, W. Zwerger, and T. J. Kippenberg, Theory of Ground State Cooling of a Mechanical Oscillator Using Dynamical Backaction, Phys. Rev. Lett. 99, 093901 (2007).

[54] M. Aspelmeyer, T. J. Kippenberg, and F. Marquardt, Cavity optomechanics, Rev. Mod. Phys. 86, 1391 (2014).

[55] M.-S. Ding, L. Zheng, and C. Li, Ground-state cooling of a magnomechanical resonator induced by magnetic damping, J. Opt. Soc. Am. B 37, 627 (2020). 